\title{
LA CIUDAD EUROPEA COMO CONCEPTO TRANSDISCIPLINARIO: CONTRADICCIONES Y POTENCIALES
}

\author{
Florian Koch \\ Instituto de Estudios Europeos \\ Universidad del Norte. Barranquilla. Colombia \\ fkoch@uninorte.edu.co
}

\section{RESUMEN}

En este artículo sostengo que el modelo de la Ciudad Europea sólo puede ofrecer una perspectiva valiosa para el desarrollo urbano y los análisis geográficos si se considera como un concepto holístico que combina diferentes disciplinas como la sociología, las ciencias políticas o el diseño urbano. Además, el concepto necesita ajustes, ya que sólo da respuestas limitadas sobre actuales desafíos urbanos como migración o cambios demográficos y por lo tanto, promueve un concepto que mira hacia atrás, que no ayuda a superar los problemas de ciudades en Europa y en otros lugares del mundo.

Palabras clave: Ciudad Europea, Desarrollo Urbano, Teoría Urbana.

\begin{abstract}
In this article I argue that the model of the European City can only provide a valuable perspective for urban development and geographical analyses if it is seen as an holistic concept which combines different disciplines like sociology, political sciences or urban design. In addition the concept needs adjustments, as it gives only limited answers to actual urban challenges like migration or demographic changes and promotes therefore a backward-looking concept which does not help to overcome the problems of cities in Europe and elsewhere.
\end{abstract}

Key words: European City, Urban Development, Urban Theory.

Fecha de recepción: diciembre 2012.

Fecha de aceptación: octubre 2013. 


\section{INTRODUCCIÓN}

La Ciudad Europea ${ }^{1}$ como un modelo para el desarrollo urbano parece ser más atractivo que nunca. Municipios y Promotores privados no sólo en Europa sino también en Asia o América Latina tratan de implementar estrategias que puedan ser englobadas bajo la etiqueta «Ciudad Europea». A pesar de su amplia distribución la definición del término es difusa y disciplinas como sociología, ciencias políticas o diseño urbano tienen su propia visión de este modelo.

Por ende, el objetivo de este artículo es examinar las diferentes conceptos de la «ciudad europea» y delinear la complejidad, las dimensiones, las contradicciones y el potencial de este modelo, así como, en particular, las dificultades que se derivan de su empleo en tiempos caracterizados por la heterogeneidad social, un papel diferente de la política urbana y una diferenciación de los desarrollos espaciales. Desde el punto de vista metodológico se analizarán aquí fuentes de diversas disciplinas y cómo comprenden el concepto de la «ciudad europea» para establecer una comparación con tendencias importantes del desarrollo urbano. Adicionalmente se hará un acercamiento a los diferentes usos del concepto.

El impulso que dio origen a la búsqueda de las dimensiones de la ciudad europea parte de la observación de que el concepto de la «ciudad europea» no se usa sólo para indicar la ubicación física de las ciudades que se encuentran en el territorio -como quiera que se sea definido- delimitado geográficamente y llamado «Europa», sino que, más bien, denomina a grandes rasgos una determinada noción de desarrollo urbano, o sea una determinada noción de ciudad. De aquí emerge la situación paradójica de que no existe una relación directa entre las ciudades del continente europeo y el concepto de la «ciudad europea» y, al mismo tiempo, el concepto de la «ciudad europea» podría aplicarse también, por ejemplo, a ciudades latinoamericanas o asiáticas. Esto evidencia que cuando se usa el concepto no se trata de nivelar las diferencias entre las ciudades europeas para abarcarlas en un modelo general aplicable a todas las ciudades de Europa, sino que se trata de un modelo ideal abstracto al cual se pueden aproximar las ciudades de todos los continentes, o no. Cabe aquí destacar que el concepto no sólo puede describir una determinada forma física de la ciudad, sino también una forma especial del desarrollo urbano en el sentido social o histórico-cultural. Por tanto, la «ciudad europea» no es un modelo urbano geográfico, como lo definieran, p. ej., Borsdorf (2003) o Mertins (2003) aplicado a las ciudades latinoamericanas, sino un concepto complejo que puede subdividirse en diferentes aspectos temáticos y funcionales y que puede tener diferentes significados en la investigación urbana y la planificación urbanística.

\section{SUBDIVISIÓN TEMÁTICA: ¿QUÉ SE ENTIENDE POR «CIUDAD EUROPEA»?}

El concepto de la «ciudad europea» se emplea en diferentes disciplinas. A continuación se presentarán los diferentes significados del concepto de la «ciudad europea» desde las perspectivas de la historiografía, las ciencias sociales, la urbanística y las ciencias políticas (cf. Frey y Koch, 2010).

1 Este artículo se basa en los resultados de un proyecto sobre el futuro de la ciudad europea que fue realizado entre 2009 y 2011 por el autor en cooperación con Oliver Frey de la Universidad Técnica de Viena, Austria. Resultados preliminares de este proyecto fueron publicados en alemán en Frey/Koch 2010, Frey/Koch 2011, y presentados por el autor en una conferencia sobre el Urbanismo europeo el 27 de junio 2011 en la Universidad Técnica de Viena. 


\section{II.1.Dimensión histórica}

En su estudio sobre la ciudad en la historia europea Leonardo Benevolo (1999, p. 13) describe las ciudades «como una de las causas - quizás la principal- de que Europa se manifieste como una unidad histórica». Y añade que la coexistencia de la mano estatal y la mano privada, característica de las ciudades europeas, que se reparten el derecho a la tierra en las ciudades, es un tema central en la historia europea, o sea, la creación de una relación equilibrada entre el derecho individual y el control público, que sólo puede funcionar allí donde los intereses de ambas partes estén representados de manera adecuada (Benevolo, 1999, p. 223). Este conflicto entre lo privado y lo público, pero también la influencia de las diferentes épocas históricas, marcaron y marcan el desarrollo urbano de las ciudades europeas y conducen a que se reconozca su «presencia» en las ciudades europeas. Aquí cuentan, entre otros elementos, la herencia del dominio feudal y las destrucciones de las guerras. Además, esta presencia histórica en las ciudades europeas es resultado de intervenciones de los gobernantes de tiempos anteriores, tanto en la estructura física de las ciudades como en la sociedad urbana. Por ejemplo, las infraestructuras de bienestar fueron establecidas en varias ciudades europeas a nivel municipal desde el siglo VIII y desempeñan hasta hoy día un papel importante en la estructura y la sociedad urbana. Como consecuencia de esto la «presencia de la historia» (Siebel, 2004, p. 18) se hace evidente y visible en la vida cotidiana de los ciudadanos. Siebel agrega que la ciudad europea es el lugar donde emerge un modo de vida especial que diferencia al ciudadano del aldeano. Ya a principios del siglo XX Georg Simmel describió al ciudadano europeo (o sea una persona que vive en una gran ciudad) como un ser de carácter reservado, insolente e intelectualizado, cualidades estas atribuibles a una reacción ante la densidad, la heterogeneidad y la diferencia de la ciudad europea (Simmel, 1986 [1903]). Sin embargo, cabría preguntar críticamente si esta diferenciación entre el espacio urbano y el espacio rural existe aún hoy en día en tal medida o si no estamos, más bien, en presencia de una urbanización de toda la sociedad.

\section{II.2.Dimensión social}

Los procesos de transformación social que tienen lugar hoy en las ciudades europeas conducen a una pluralización y a una heterogeneización de los mundos sociales. Las sociedades urbanas de la actualidad producen una gran variedad de medios sociales: El medio social urbano de la burguesía clásica, el de la escena verde-alternativa, el de los trabajadores o el de las subculturas juveniles se diferencias marcadamente entre sí y conducen a que los individuos se sientan más alienados en su vida diaria (cf. Frey, 2012). Caracterizar a la ciudad europea como un «mosaico de mundos sociales» significa que la ciudad no conforma una unidad social-espacial, sino que es un espacio urbano estructurado por espacios sociales separados entre sí y que no se mezclan. Pero aquí hay que aclarar que la alienación y la diferenciación de los medios sociales urbanos no es una característica exclusiva de las ciudades europeas, sino que también pueden caracterizar a, por ejemplo, ciudades americanas o asiáticas.

Sin embargo, la ciudad europea como formación social se caracteriza por un menor grado de segregación espacial-social, sobre todo en comparación con las ciudades en Estados Uni- 
dos (cf. Bagnasco y Le Galès, 2000, p. 14). Al mismo tiempo se considera el papel de los ciudadanos como actores activos que modelan la vida de la ciudad con su participación en, p. ej., organizaciones, sociedades, federaciones, iniciativas, etc., como una característica de la ciudad europea. El desarrollo de la ciudad, por ende, no está determinado sólo por los representantes elegidos u otros actores públicos, sino por una gran variedad de los más disímiles grupos y microproyectos que en su minoría cuentan con una forma institucional (Le Galès, 2002, p. 262).

\section{III.3. Dimensión política}

La dimensión política de la ciudad europea resulta de su inserción en un estado nacional del bienestar social y sus amplias libertades de acción a nivel comunal dentro de la organización nacional. Esto significa que las ciudades y las comunas reciben fondos nacionales y, con ellos, pueden determinar hasta cierto punto su política y su desarrollo dentro de los límites establecidos (cf. Kazepov, 2005). También la inserción en el sistema supranacional de la Unión Europea aumenta la libertad para las ciudades europeas porque además de los fondos nacionales, las ciudades pueden buscar fondos de la Unión Europea en los programas de fomento europeos. Con algunas restricciones esto aplica también para las ciudades en países europeos que no son miembros de la Unión Europea. En el modelo opuesto de las ciudades estadounidenses la ciudad tiene menos libertad de acción, ya que recibe menos fondos nacionales/supranacionales y, por lo tanto, tienen que actuar mucho más como actores del mercado. De aquí resulta una gran influencia de la mano estatal (local) que se evidencia en intervenciones concretas, como p. ej. las inversiones físicas en la infraestructura, el abastecimiento energético y de agua, las tierras comunales, el control del mercado de la vivienda y, sobre todo, el urbanismo.

En las ciudades europeas el urbanismo moderno se desarrolló a partir de la mitad del siglo XIX. Aquí surgió una caja de herramientas urbanísticas conformadas por instrumentos formales e informales que posibilitó «domar» las fuerzas del mercado y, con ello, un desarrollo espacial ordenado, controlado por los actores públicos (cf. Koch, 2011).

\section{III.4. Dimensión urbanística}

Marcuse (2004, p. 112) menciona como características de la ciudad europea un centro histórico con edificaciones bajas -a excepción de las religiosas y/o estatales-, con plazas públicas centrales, barrios de estructura social mixta con comercios pequeños, límites geográficos claros, una gran densidad de población y de urbanización y un sistema de transporte público denso. Urban (2008) también menciona la mezcla densa de las funciones y trato sensible de las edificaciones históricas entre las características de la ciudad europea. El espacio público, sobre todo la plaza del mercado, tiene una gran importancia aquí por ser el lugar democrático donde se forma la sociedad urbana en las ciudades medievales de Europa, donde confluyen los diferentes grupos y capas sociales (Hassenpflug, 2002, cf. Farías, 2005). Precisamente el diseño del espacio público y su uso por parte de diferentes grupos sociales se considera una gran diferencia entre la ciudad europea y, p. ej., la ciudad latinoamericana, que desde su fundación por los españoles tuvo espacios públicos vedados para determinados grupos sociales 
(Flores, 2004, p. 137) y donde la exclusión y la fragmentación siguen siendo consideradas hasta hoy una característica esencial del modelo de la ciudad latinoamericana (Janoschka, 2002). Sin embargo, la ciudad europea fue por largo tiempo menos abierta y democrática de lo que muchas veces se asume. Hasta hace poco sólo determinados grupos sociales tenían la posibilidad de participar en las decisiones políticas y gozar de derechos ciudadanos. Una gran parte de la población (personas sin posesión de tierra o mujeres) se encontraban fuera de la participación municipal y no tenían derecho a votar. Por ejemplo, en la Alemania de inicio del siglo XIX sólo 20 por ciento de la población urbana tenía plenos derechos ciudadanos (cf. Koch, 2010, p. 30; Wehling, 2006, p. 10). También el acceso al espacio público estaba restringido. A pesar de la noción, muchas veces romantizada, de la ciudad europea como ciudad abierta, se caracteriza su estructura urbana con términos como densa, compacta y central. Por eso Farias (2005) considera el programa de fomento de la Unión Europea, de 1975, para proteger el legado arquitectónico de los centros y plazas del mercado medievales como el momento en que los europeos redescubrieron la ciudad europea.

La objeción justificada de que en las ciudades europeas existen desarrollos opuestos a estas cualidades, como ciudades intermedias y centros despoblados, y de que no existe una forma de ciudad predominante en todo el continente europeo, demuestra una vez más que el concepto de la «ciudad europea» no es de naturaleza descriptiva, o sea, que describe un modelo de desarrollo urbano real, sino que se trata de un modelo ideal.

Por consiguiente, se puede concluir que no existe un modelo de ciudad europea, sino diferentes definiciones de lo que constituye a la ciudad europea según la disciplina que emplea la definición. No obstante, existen puntos en común entre los contenidos de las diferentes disciplinas, por ejemplo, los instrumentos políticos del urbanismo condicionan la forma urbana de la ciudad europea.

\section{SUBDIVISIÓN FUNCIONAL: ¿PARA QUÉ SE USA EL CONCEPTO DE LA «CIUDAD EUROPEA»?}

Además de sus significados temáticos el concepto de la «ciudad europea» tiene también diferentes connotaciones funcionales. Por un lado se emplea como modelo normativo-utópico, pero por otro también como marco analítico de desarrollos urbanísticos y de la política y la sociedad urbanas. Entre ambas funciones existen puntos en común que muchas veces dificultan su clara delimitación (cf. Häußermann y Haila, 2005).

\section{III.1. Marco analítico}

El empleo analítico del concepto se remonta al sociólogo alemán Max Weber (2000), quien definió cinco características de la ciudad europea medieval que, según él, fueron la causa de que surgieran formas de trabajo capitalistas precisamente en las ciudades europeas: Una fortificación, un mercado, un código legal y cortes de justicia propias, una asociación de ciudadanos que creó una suerte de corporación municipal, y suficiente autoadministración comunal como para que los ciudadanos pudieran elegir a sus gobernantes.

Por supuesto no todos los aspectos nombrados por Weber caracterizan a la ciudad europea de la actualidad, como p. ej. la jurisdicción propia y la delimitación física por los muros de la ciudad, pero su enfoque de nombrar las particularidades de la ciudad y su 
énfasis en la autoadministración comunal, siguen encontrando aplicación en los llamados enfoques neoweberianos de hoy. Dos de sus representantes, Bagnasco y Le Galès (2000), consideran la formación de la sociedad urbana como actor colectivo o formación social, que resulta de la interacción de diferentes grupos y organizaciones sociales, como una característica de la ciudad europea. En su calidad de marco analítico las diferentes características pueden usarse para comparar procesos urbanos de diferentes ciudades y examinar qué tan «europea» es una ciudad (cf. p. ej. los análisis de Giersig, 2008 sobre Estocolmo y Helsinki o de Betz, 2011 sobre la Cuenca del Ruhr). El marco analítico puede contener los elementos políticos, urbanísticos, sociales e históricos de la ciudad europea descritos en la sección anterior y analizar el desarrollo real de diferentes ciudades a través de estos criterios.

Este intento de definir las características de la ciudad europea que hacen posible diferenciarla de otras ciudades es opuesto al enfoque de las teorías urbanas universalistas (cf. Giersig, 2008), que parten de que el desarrollo urbano se homogeneiza cada vez más a nivel mundial, p. ej. como consecuencia de la globalización (cf. entre otros Sassen, 1991; Castells, 2000). Sin embargo, con ayuda del marco analítico «ciudad europea» se puede mostrar diferencias y matices en los desarrollos. A la ciudad europea se contrapone a menudo la ciudad norteamericana, que se describe con características mayormente negativas, como crecimiento urbano descontrolado, ausencia de planificación, amasijo urbano, marcada segregación social y centros despoblados. Aquí no se abordará la cuestión de en qué medida esta comparación de modelos blanco y negro se corresponde con la realidad (para ello cf. Hannemann y Mettenberger, 2011; Keil 1999), pero esta comparación pone en evidencia la función normativa que posee el marco analítico «ciudad europea» en muchas ocasiones, de manera implícita o explícita.

\section{III.2. El modelo normativo-utópico}

El modelo normativo-utópico ve la ciudad europea como modelo positivo de un desarrollo urbano con futuro por el cual se deben orientar las ciudades. Tómese como ejemplo un informe actual sobre el desarrollo urbano del Gobierno alemán, donde el modelo normativoutópico de la «ciudad europea» tiene un papel destacado: «El punto de partida y el modelo a seguir de todas las medidas y actividades urbanísticas del Gobierno alemán es la 'ciudad europea', concebida como un modelo de organización del espacio, de vida social y de valores, pero también como un modelo de éxito. [...] La misma es el modelo de urbanización adecuado para apoyar la prosperidad económica, así como la integración social, y además es sensata desde el punto de vista ecológico» (BMVBS, 2009). En este ejemplo la ciudad europea no se reduce solamente a los criterios urbanísticos de la densidad, la compacidad y la centralidad, sino que incluye aspectos como el crecimiento económico y el equilibrio social. En otros contextos se considera sobre todo la dimensión urbanística de la ciudad europea como modelo a seguir; ejemplo de esto son las urbanizaciones del New Urbanism en Estados Unidos, las ciudades planeadas en China o las comunidades cerradas de Latinoamérica o Europa del Este, siendo estas últimas aquellas que siguen el modelo del New Urbanism y copian así algunos de los elementos urbanísticos de la ciudad europea pero generalmente sin tener en cuenta la dimensión social, política e histórica. 
El intento aquí realizado de describir la ciudad europea a través de sus diferentes perspectivas temáticas y funcionales demuestra, por una parte, lo diverso que es este concepto, pero también, por otra parte, las dificultades que se derivan de su empleo, pues con frecuencia funciona como una base que puede servir para apuntalar las más disímiles ideas y conclusiones. A la vista de las variadas facetas de este concepto no es de extrañar que apelando a la ciudad europea se puedan realizar proyectos de New Urbanism, apoyar la implementación de una protección de monumentos estricta y la reconstrucción de edificaciones históricas o realizar estudios e intervenciones para reducir los procesos de polarización o de privatización de los espacios públicos. En ninguno de estos casos la referencia sería incorrecta, pues, evidentemente, la ciudad europea se presta como marco de referencia para casi cualquier forma de desarrollo urbano gracias a sus diferentes características.

\section{CONTRADICCIONES DEL MODELO DE LA «CIUDAD EUROPEA»}

Hay que señalar que si bien los diferentes significados que se asocian al concepto de la «ciudad europea» demuestran lo variado que es el modelo, en última instancia también se pueden contradecir entre sí y conducir a problemas a la hora de emplearlo. Häußermann (2001) indicó que la gran libertad de acción de la política comunal -una de las características de la ciudad europea- condujo a que las estructuras arquitectónicas de la ciudad europea medieval fueran destruidas por las medidas de saneamiento drásticas de las décadas del ‘60 y '70 del siglo pasado. Las intervenciones públicas y el instrumentario urbanístico correspondiente llevaron a los saneamientos a gran escala que destruyeron las estructuras arquitectónicas típicas de la ciudad europea, como las manzanas cerradas y las estructuras mixtas con pequeños comercios. Precisamente por ser la ciudad europea un actor con gran libertad de acción es que se pudieron realizar proyectos de esta envergadura e índole opuesta a la tradición urbanística de la ciudad europea.

Otra característica de la ciudad europea, la fuerte influencia de sus ciudadanos en el desarrollo urbano, también puede conducir en última instancia a una mayor polarización de la sociedad urbana; cuando agrupaciones de ciudadanos deciden poner cámaras y personal de vigilancia en sus barrios para excluir a sectores de la población indeseados, pueden poner en marcha procesos de segregación.

También resultan contradictorias las «migraciones» del modelo de la «ciudad europea». Los principios urbanísticos de las ciudades europeas se tomaron como modelo para fundar ciudades en Latinoamérica, Asia o África, pero bajo el signo del colonialismo se segregó a la población indígena, por lo que se asumieron sólo los modelos urbanísticos formales de la ciudad europea, obviando los sociales. También en el siglo XXI la «ciudad europea» es un codiciado «artículo de exportación» (Urban, 2008). En China, por ejemplo, se construyen nuevos barrios y ciudades enteras según los principios urbanísticos europeos, como la densidad y la compacidad arquitectónica y el uso mixto. Por estos principios se orientan igualmente los proyectos del New Urbanism como la urbanización Seaside en Florida, Estados Unidos. La realización física de estos proyectos ha sido más o menos exitosa, pero es evidente que es mucho más difícil transferir otros aspectos de la ciudad europea, como p. ej. la introducción de la libertad de acción o el compromiso social de sus ciudadanos. Se 
puede poner muy en duda que un caparazón arquitectónico denso y compacto, con la reluciente etiqueta de «europeo», pueda ser llenado con los atributos de una «ciudad europea». A modo de conclusión se puede afirmar que la «ciudad europea» es un artículo de exportación, sin lugar a dudas exitoso, como modelo urbanístico predominantemente formal, pero que sus realizadores descuidan otros aspectos.

\section{LA «CIUDAD EUROPEA»: ¿UN MODELO URBANÍSTICO PARA EL FUTURO DENTRO Y AFUERA DE EUROPA?}

A pesar de las contradicciones mencionadas anteriormente la «ciudad europea» goza de una gran aceptación como modelo urbanístico. Por regla general sus seguidores toman algunas de las características temáticas de la ciudad europea, anteriormente mencionadas, como directrices normativas y aspiran a lograr un desarrollo urbano que se orienta desde el punto de vista político, urbanístico y social por el concepto de la «ciudad europea». Pero se debe analizar si estas características típicas e ideales sirven hoy en día todavía como orientación para el desarrollo urbano real en las ciudades dentro y fuera de Europa. En otras palabras: ¿Se ha alejado tanto la realidad del desarrollo urbano del modelo de la ciudad europea, que seguir aferrándose a este modelo resulta más bien un obstáculo, en lugar de servir para controlar los desarrollos futuros de manera adecuada y que conduzca al objetivo propuesto? A continuación se comprobará esto a partir de las tendencias actuales del desarrollo en las ciudades europeas y con ayuda de las dimensiones temáticas.

La dimensión política de la ciudad europea, la amplia libertad de acción comunal, está perdiendo importancia. Es un hecho que actualmente la política comunal ya no puede controlar muchos aspectos esenciales del desarrollo espacial y que eso exige nuevos enfoques de gobernabilidad regional. Por consiguiente, el llamado a contar con un actor comunal fuerte y una extensa planificación comunal -una de las características descritas de la ciudad europea- no tiene en cuenta la realidad y los retos futuros de la administración local y regional. Muchos desarrollos esenciales ya no tienen lugar dentro de los límites geográficos de la ciudad, sino en la conurbación. Por lo tanto, el modelo de la ciudad europea -que parte de que la unidad política «ciudad» debe poseer amplias posibilidades de administración para controlar autónomamente el desarrollo dentro de sus límites- no es adecuado para la conurbación policentral y la cooperación intercomunal.

Un ejemplo de la incapacidad de las ciudades para asumir nuevos retos es el cambio climático. Las ciudades tienen que adaptarse e implementar medidas adecuadas. Sin embargo en vista de la complejidad de la tarea y el impacto del cambio climático no solo dentro de las fronteras municipales, las regiones se convierten en actores potentes para manejar la adaptación y no los municipios (Birkmann et al. 2013).

En la dimensión social se reconoce cada vez menos a la sociedad urbana que contribuye activamente como actor o formación social al desarrollo de su ciudad, lo cual se opone a las características de la ciudad europea. Los procesos de migración e integración cambian las sociedades urbanas. A causa del mayor número de hogares multilocales o de migrantes sin estatus legal resulta evidente que cada vez se hace más difícil definir la pertenencia de estas personas a una sociedad urbana. Los hogares multilocales pueden componerse de personas que viven en una ciudad y trabajan en otra, así que trabajan durante la semana en 
una ciudad y regresan a sus casas los fines de semana y, por tanto, es difícil definir claramente su pertenencia a un solo lugar. Por otra parte existen en muchas ciudades europeas formas de vida transnacionales, es decir inmigrantes que tienen un vínculo más fuerte con sus comunidades originarias que con la sociedad donde viven y trabajan. Un ejemplo de esto son los inmigrantes latinoamericanos en Europa, que transfieren dinero regularmente a sus países de origen y de esta manera tienen una influencia directa y notable en su desarrollo espacial y social, mientras que en las ciudades europeas donde trabajan están menos presentes. Debido a los procesos de la globalización, las transformaciones en las estructuras familiares y las condiciones de trabajo de estos hogares multilocales y transnacionales han cobrado mayor importancia. Teniendo en cuenta esta realidad de la vida, los procesos clásicos de participación y compromiso ciudadano de las personas y las instituciones sociales con el desarrollo de «su ciudad»-una de las características descritas de la ciudad europea- sólo pueden tomarse con restricciones como modelo de desarrollo urbano.

También la dimensión urbanística de la ciudad europea debe seguir evolucionando como modelo en un nuevo contexto. Si bien la densidad y compacidad y el uso mixto pueden ser adecuadas como directrices para la construcción de nuevas urbanizaciones, en la realidad los urbanistas en las ciudades europeas se ven enfrascados cada vez más en mejorar las estructuras arquitectónicas existentes. Otra tendencia importante en las ciudades europeas son los procesos de contracción, como consecuencia de la reducción de la población, y sus viviendas, inmuebles comerciales e instalaciones de la infraestructura que quedan vacíos y, por tanto, se vuelven innecesarios. En las ciudades en contracción la cuestión primaria ya no es sumar nuevas estructuras arquitectónicas a las existentes, sino más bien desmantelar edificaciones superfluas y dar respuesta a la pregunta de cómo lograr que las ciudades sigan funcionando bien y sean vivibles a pesar de que su población decrece. En este sentido es de esperar que nuevos modelos y enfoques jueguen un papel más importante en este contexto que la forma tradicional de la ciudad europea. De hecho, en los últimos años ha surgido ya un debate sobre nuevos modelos como la «ciudad perforada», «contracción de afuera hacia adentro» y «uso temporal del espacio».

La transferencia del modelo de la «ciudad europea» en otros continentes especialmente en países en vía de desarrollo se debe mirar críticamente. Está en duda que el modelo sea útil para los desafíos actuales que existen en muchas ciudades asiáticas, africanas o latinoamericanas. Por ejemplo, aspectos como infraestructura insuficiente, el alto grado de privatización de bienes públicos y ligado a esto la desigualdad de oportunidades, la alta informalización del mundo laboral, las altas tasas de criminalidad y el limitado funcionamiento estatal no encuentran en las características clásicas de la «ciudad europea» una guía que responda a la superación de estas necesidades y más bien requieren reorientarse hacia otros caminos.

\section{CONCLUSIONES}

El análisis anterior permite concluir que la «ciudad europea» no es adecuada como modelo futuro para el urbanismo y que es un mito que ya no es capaz de describir el desarrollo real de las ciudades europeas. Cuando se intenta emplear las características de la «ciudad europea» como modelo normativo se choca rápidamente con sus límites. Las condiciones 
económicas, sociales y ecológicas han cambiado tanto que la «ciudad europea» no parece adecuada como esbozo concreto del futuro o como consenso de planeación urbanística para lograr los objetivos específicos del urbanismo.

Sin embargo, la ciudad europea alberga una particularidad importante, por la cual sigue funcionando como modelo actual para el desarrollo urbano, y es que, como ya se expuso anteriormente, este concepto sólo puede entenderse en el contexto de la interacción entre sus dimensiones arquitectónicas, urbanísticas, sociales, históricas y políticas. Las perspectivas unidimensionales, como p. ej. las que se concentran en la forma física de la ciudad europea, dejan de lado otros aspectos esenciales y no sirven ni de marco analítico ni de modelo político-normativo.

No obstante estos puntos de crítica, el concepto de la «ciudad europea» tiene todavía su importancia y su futuro. El modelo ofrece la oportunidad de abarcar la compleja realidad de las ciudades de manera interdisciplinaria. Como modelo para el urbanismo esto representa la posibilidad de formular amplias exigencias para el desarrollo urbano que se pueden llevar a la práctica sólo en cooperación con varias disciplinas. Si se asume el concepto de la ciudad europea menos como un manual de instrucciones para el desarrollo urbano y más como la posibilidad de aunar los esfuerzos de las ciencias sociales, la política, la historiografía y el urbanismo, se tendrá entonces una nueva visión de lo urbano que puede resultar muy fructífera no sólo para las ciudades europeas.

Es necesario seguir desarrollando las tipologías y las características de la «ciudad europea» con ayuda de los cambios actuales de la realidad urbana expuestos anteriormente. Este desarrollo puede conducir a diferentes interpretaciones del modelo de la «ciudad europea» en función de la ciudad (conurbación) en cuestión. Pero la perspectiva interdisciplinaria de las estructuras arquitectónicas-espaciales y su uso, su formación social y su estructuración social con los procesos de control y los instrumentos y métodos de planificación de la política urbana será siempre semejante allí donde el concepto se emplee no sólo como un eslogan vacío.

\section{REFERENCIAS}

BAGNASCO, A., \& LE GALÈS, P. (2000): Cities in contemporary Europe. Paris: CEVIPOF.

BENEVOLO, L. (1999). Die Stadt in der europäischen Geschichte. München: Europa Bauen.

BETZ, G. (2011): Ruhrgebiet - europäische Stadt im Werden? Strukturwandel und Governance durch die Kulturhauptstadt Europas RUHR.2010. En Frey/Koch, Die Zukunft der Europäischen Stadt. Stadtpolitik, Stadtplanung und Stadtgesellschaft im Wandel. Wiesbaden: VS-Verlag für Sozialwissenschaft, 324-342.

BIRKMANN, J., VOLLMER, M. y SCHANZE, J. (2013): Raumentwicklung im Klimawandel. Herausforderungen für die räumliche Planung. Hannover: ARL.

BMVBS [BUNDESMINISTERIUM FÜR VERKEHR, BAU UND STADTENTWICKLUNG]. (15 de Septiembre de 2009): Stadtentwicklungsbericht 2008. Neue urbane Lebens- und Handlungsräume. Obtenido de http://www.bmvbs.de/Anlage/original_1075468/Stadtentwicklungsbericht-derBundesregierung-2008.pdf 
BORSDORF, A. (2003): «Cómo modelar el desarrollo y la dinámica de la ciudad latinoamericana». EURE, vol. 29, n 86.

CASTELLS, M. (2000): «The space of flows». En M. Castells, \& I. Susser, The Castells reader on cities and social theory. Oxford: Blackwell, 314-360.

FARÍAS, I. (2005): «A la búsqueda del «Urbanismo Europeo: un reporte desde Berlín». EURE, vol.31, $\mathrm{n}^{\circ} 94$.

FLORES, P. (2004): «La ciudad Europea o los desplazamientos del centro». En J. Ferro Bayona, C. H. de Peña, \& J. Villalón, Memoria Catedra Europa 2003. Barranquilla: Ediciones Uninorte.

FREY, O. (2012): »Städtische Milieus». En F. Eckardt, Handbuch Stadtsoziologie. Wiesbaden: VS Verlag für Sozialwissenschaften.

FREY, O., \& KOCH, F. (2010): Die Europäische Stadt. Dimensionen und Widersprüche eines transdisziplinären Leitbilds. Raumplanung 153, 261-266).

FREY, O., \& KOCH, F. (2011): Die Zukunft der europäischen Stadt. Wiesbaden: VS Verlag für Sozialwissenschaften .

GIERSIG, N. (2008): Urban Governance and the 'European City'. Discussing Metropolitan Reforms in Stockholm and Helsinki. Wiesbaden: VS Verlag für Sozialwissenschaften.

HANNEMANN, C., \& METTENBERGER, T. (2011). Zur Funktion einer konzeptionell orientierten Dualität. En O. Frey, \& F. Koch, Die Zukunft der europäischen Stadt. Wiesbaden: VS Verlag für Sozialwissenschaften, 55-70.

HASSENPFLUG, D. (2002): Die europäische Stadt als Erinnerung, Leitbild und Fiktion. En D. Hassenpflug, Die Europäische Stadt -Mythos und Wirklichkeit. Münster-HamburgLondon : LIT Verlag.

HÄUSSERMANN, H. (2001): Die europäische Stadt». Leviathan 29, 237-255.

HÄUSSERMANN, H., \& HAILA, A. (2005): The European City: A Conceptual Framework and Normative Project. . En Y. Kazepov, The European City: A Conceptual Framework and Normative Project, in Cities of Europe: Changing Contexts, Local Arrangements, and the Challenge to Urban Cohesion. Oxford: Blackwell Publishing Ltd, 43-63.

JANOSCHKA, M. (2002): El nuevo modelo de la ciudad latinoamericana: fragmentación y privaticacion. Eure, Vol.28, No. 85 .

KAZEPOV, Y. (2005): Changing Contexts, local arrangements and the challenge to Urban cohesion. En Y. Kazepov, Cities of Europe. Oxford: Blackwell, 3-42.

KAZEPOV, Y. (2005): Cities of Europe. Oxford: Blackwell.

KEIL, R. (1999): Die Entdeckung Amerikas in der Stadt. En W. Prigge, Peripherie ist überall. Frankfurt a. M.; New York.

KOCH, F. (2010): Die europäische Stadt in Transformation. Stadtentwicklungspolitik und Stadtplanung im post-sozialistischen Warschau. Wiesbaden: VS Verlag für Sozialwissenschaften.

KOCH, F. (2011): Stadtplanung, Governance und Informalität: Vorschlag einer Typologie. En Frey/Koch, Die Zukunft der europäischen Stadt. (págs. 191-207). Wiesbaden: VS Verlag für Sozialwissenschaften.

LE GALÈS, P. (2002): European cities. Social conflicts and governance. Oxford: Oxford University Press.

MARCUSE, P. (2004): Verschwindet die europäische Stadt in einem allgemeinen Typus der globalisierten Stadt? En W. Siebel, Die europäische Stadt. Frankfurt a. M; 112-117. 
MERTINS, G. (2003): Transformaciones recientes en las metrópolis latinoamericanas y repercusiones espaciales. En J. Luzón, C. Stadenl, \& C. Borges, Transformaciones regionales y urbanas en Europa y América Latina. Barcelona: Publicaciones de la Universidad de Barcelona, 191-207.

SASSEN, S. (1991): The Global City: New York/London/Tokyo. Princeton: Princeton University Press.

SIEBEL, W. (2004): Die europäische Stadt. Frankfurt a. M.: Suhrkamp Verlag.

SIMMEL, G. (1986 [1903]): Las grandes urbes y la vida del espíritu. En G. Simmel, El individuo y la libertad. Ensayos de crítica de la cultura. Barcelona: Ediciones Península, $247-261$.

URBAN, F. (2008): Ciudad del futuro. La Ciudad Europea Global. Obtenido de Instituto Goethe: http://www.goethe.de/kue/arc/dos/dos/sls/sdz/es3481742.htm

WEBER, M. (2000): Wirtschaft und Gesellschaft. Die Stadt. Studienausgabe der MaxWeber-Gesamtausgabe Band I/22-5. Tübingen: J.C.B. Mohr.

WEHLING, H.-G. (2006): Kommunen früher und heute. Informationen zur politischen Bildung, Nr. 242/2006 : Kommunalpolitik , 7-27. 